\title{
RESPOSTA DE MUDAS DE Senna macranthera (DC. EX COLLAD.) H.S. IRWIN \& BARNABY (FEDEGOSO) CULTIVADAS EM LATOSSOLO VERMELHO- AMARELO DISTRÓFICO A MACRONUTRIENTES ${ }^{1}$
}

\author{
Cezar Augusto Fonseca e Cruz², Haroldo Nogueira de Paiva ${ }^{3}$, Julio Cesar Lima Neves ${ }^{4}$ e \\ Ana Catarina Monteiro Carvalho Mori da Cunha ${ }^{5}$
}

\begin{abstract}
RESUMO - Senna macranthera (Fedegoso) tem grande ocorrência natural e potencial para uso em recuperação de áreas degradadas. Dada a grande necessidade de informações sobre a nutrição de espécies florestais a exemplo da citada, avaliou-se a resposta das plantas de Senna macranthera a doses de N, P, K, Ca, Mg e S sobre o crescimento e produção de biomassa, bem como sobre os índices de qualidade de mudas H/D, H/MSPA, MSPA/ MSR e IQD. O trabalho, conduzido em casa de vegetação, utilizou amostras de um Latossolo Vermelho- Amarelo distrófico retiradas da camada abaixo de $20 \mathrm{~cm}$, secas ao ar, peneiradas e caracterizadas quimicamente. Delimitouse o trabalho por meio de uma matriz baconiana, avaliando três doses dos seis macronutrientes e dois tratamentos adicionais, um com doses de referência e outro sem adição de nutrientes. Adotou-se o delineamento inteiramente casualizado com quatro repetições, num total de 80 unidades amostrais. Colhidas as plantas, verificou-se, através da análise dos contrastes ortogonais, resposta significativa aos tratamentos aplicados a todas as variáveis estudadas. Não foi possível determinar a dose crítica para todos os nutrientes em todas as diferentes variáveis analisadas. Verificou-se, também, a necessidade de maior diferenciação entre as doses aplicadas, bem como de adoção de um tratamento-testemunha por nutriente. É interessante aumentar o tempo experimental, tendo em vista o crescimento mais lento observado da espécie, comparativamente a outras já estudadas.
\end{abstract}

Palavras-chave: Dose crítica de macronutrientes, Nutrição de plantas e Produção de mudas.

\section{RESPONSE OF Senna macranthera (DC. EX COLLAD.) H.S. IRWIN\&BARNABY (FEDEGOSO) SEEDLINGS PRODUCED IN A DYSTROPHIC RED-YELLOW LATOSOL TO MACRONUTRIENTS}

\begin{abstract}
Senna macranthera (Fedegoso) has large natural occurrence, with potential use in the recovery of degraded areas. Due the lack of information on the nutrition of this forest species, this work evaluated the response of Senna macranthera to the doses of $\mathrm{N}, \mathrm{P}, \mathrm{K}, \mathrm{Ca}, \mathrm{Mg}$ and $\mathrm{S}$ on the growth and biomass production, and the indexes of quality of seedlings H/D, H/MSPA, MSPA/MSR and IQD. The experiment was carried out in a greenhouse and used samples of a dystrophic red-yellow latosol of the layer bellow $20 \mathrm{~cm}$ that were air dried, sieved and chemically characterized. The treatments were delimited according to a Baconian matrix, with three doses of the six macronutrientes and two additional treatments, one with reference doses and other without addition of nutrients. The experiments were arranged in a complete randomized design, with 4 repetitions, in a total of 80 sample units. Plants were collected and the orthogonal contrast analysis showed significant differences for all the treatmens applied to the studied variables. In spite of that, it was not possible to determine the critical nutrient dose for all analyzed variables. It was also verified the need for a greater differentiation among the tested doses, as well as of a control treatment for each studied nutrient. It is also recommended to increase the experimental time because of the slow growth rate of this species compared with others already studied.
\end{abstract}

Keywords: Critical doses of macronutrients, Plant nutrition and Seedling production.

\footnotetext{
${ }^{1}$ Recebido em 28.05.2008 e aceito para publicação em 14.10.2009.

${ }^{2}$ Instituto Estadual de Florestas de Minas Gerais, Núcleo Operacional de Florestas, Pesca e Biodiversidade Poços de Caldas, Pocos de Caldas, MG - Brasil .E-mail: <ceaufoc1@gmail.com>.

${ }_{3}^{3}$ Departamento de Engenharia Florestal, Universidade Federal de Viçosa - Vicosa, MG - Brasil. E-mail: <hnpaiva@ufv.br>. ${ }^{4}$ Departamento de Solos, Universidade Federal de Viçosa - Vicosa, MG - Brasil. E-mail: <julio_n2003@yahoo.com.br>.

${ }^{5}$ Programa de Pós-Graduação em Ciência Florestal, Departamento de Engenharia Florestal, Universidade Federal de Viçosa.

E-mail: <catarina_mori@yahoo.com.br>.
} 


\section{INTRODUÇÃO}

Senna macranthera (fedegoso), espécie florestal aqui estudada, ocorre naturalmente dos Estados do Ceará até São Paulo, nas Florestas Semidecíduas de Altitude (LORENZI, 1992). Esse autor ressaltou, ainda, que a espécie é bastante ornamental quando em flor e, devido à sua adaptação a diversos tipos de solos e ao seu rápido crescimento, é considerada própria para utilização em arborização urbana e programas de revegetação em áreas degradadas e matas ciliares (LORENZI, 1992).

A necessidade deste estudo advém, também, de uma situação muito comum nas propriedades rurais mineiras: a degradação das pastagens, que ocorre devido ao manejo inadequado destas pelos produtores rurais (CARVALHO, 1997). De acordo com esse autor, o manejo incorreto pode ocasionar o surgimento de atividades erosivas, com consequente perda de solo e baixa retenção de água de chuva nele.

Exatamente por isso tem sido pensado o uso de espécies florestais arbóreas como forma de evitar a degradação das pastagens em áreas de topografia ondulada, paisagem comum no Estado de Minas Gerais. Tal pensamento leva em conta o grande potencial que as espécies arbóreas têm no controle da erosão, além de aumentar a disponibilidade de diversos nutrientes no solo, especialmente o nitrogênio (CARVALHO, 1997).

A determinação de doses críticas dos nutrientes em cada tipo de substrato e o relacionamento delas com a performance da planta possibilitarão uma recomendação melhor e mais segura da adubação para a produção de mudas de dada espécie. Neves (1983) afirmou que as espécies arbóreas apresentaram diferenças não apenas em suas exigências nutricionais, mas também quanto à eficiência de absorção e/,ou, utilização dos nutrientes.

Os solos a serem reflorestados são distintos quimicamente, o que, associado à grande diversidade de demanda nutricional das espécies florestais, indica a necessidade de maiores estudos visando à obtenção de informações sobre a sua nutrição e a resposta dessas plantas a um programa de fertilização (FERNANDES et al., 2000).

Este trabalho foi conduzido com o objetivo de avaliar o efeito da aplicação de doses dos macronutrientes sobre as plantas de fedegoso. Ao final do período experimental, procurou-se determinar a dose crítica desses nutrientes para a produção de mudas de fedegoso para a permitir a construção de adequado programa de adubação que permita o uso da espécie na recuperação de pastagens degradadas.

\section{MATERIAL E MÉTODOS}

\section{1. Área de estudo}

O trabalho foi conduzido em casa de vegetação no viveiro de pesquisa do Departamento de Engenharia Florestal da Universidade Federal de Viçosa, em Viçosa, MG, no período de janeiro a junho de 2006 . Viçosa situase nas coordenadas $20^{\circ} 45^{\prime} \mathrm{S}$ e $42^{\circ} 55^{\prime} \mathrm{W}$ na Zona da Mata de Minas Gerais (MARISCAL-FLORES, 1993). O clima é classificado como tropical de altitude, com verões chuvosos e invernos frios e secos, do tipo Cwb, pelo sistema de Köeppen. A precipitação média anual é de $1.221 \mathrm{~mm}$ (DNM, 1992).

A espécie escolhida foi Senna macranthera (fedegoso), sendo suas sementes adquiridas no setor de silvicultura do Departamento de Engenharia Florestal da UFV. Utilizaram-se como substrato amostras de um Latossolo Vermelho-Amarelo distrófico provenientes de áreas próximas ao Município de Viçosa. Resende et al. (1988) afirmaram que esse solo têm ocorrência predominante na região da Zona da Mata mineira, tendo sido, por isso, escolhido para a realização do experimento. As amostras foram retiradas da camada abaixo de 20 $\mathrm{cm}$ de profundidade, secas ao ar, peneiradas em malha de $4 \mathrm{~mm}$ de diâmetro e devidamente caracterizadas fisicamente (argila 60\%, silte 9\%, areia grossa $14 \%$ e areia fina $17 \%$ ) e quimicamente (Tabela 1 ).

\subsection{Tratamentos}

Para delimitar o trabalho, utilizou-se uma matriz baconiana (TURRENT, 1979), avaliando três doses de nitrogênio $(\mathrm{N})$, fósforo $(\mathrm{P})$, potássio $(\mathrm{K})$, cálcio $(\mathrm{Ca})$, magnésio (Mg) e enxofre (S) e mais dois tratamentos adicionais; um com doses de referência e outro sem adição de nutrientes. Ao todo, avaliaram-se 20 tratamentos dispostos segundo um delineamento inteiramente casualizado com quatro repetições, num total de 80 unidades amostrais.

Os tratamentos foram dispostos de forma que, quando a quantidade de um nutriente variasse, as doses dos outros estariam fixas (Tabela 2). A aplicação de N, assim como a de $\mathrm{K}$ foi parcelada e feita após a semeadura $(0,30,50,70$ e 90 dias $)$. 
Tabela 1 - Análise química das amostras de solos utilizadas na produção das mudas antes da aplicação dos tratamentos. Table 1 - Chemical analysis of soil samples used in the production of seedlings before treatment application.

\begin{tabular}{|c|c|c|c|c|c|c|c|c|c|c|c|}
\hline \multirow[t]{2}{*}{ Solo } & $\mathrm{pH}$ & $\mathrm{P}$ & $\mathrm{K}$ & \multirow[t]{2}{*}{$\mathrm{Ca}^{2+}$} & \multirow[t]{2}{*}{$\mathrm{Mg}^{2+}$} & $\mathrm{Al}^{3+}$ & $\mathrm{H}+\mathrm{Al}$ & \multirow[t]{2}{*}{ SB } & \multirow[t]{2}{*}{$(\mathrm{T})$} & \multirow{2}{*}{\multicolumn{2}{|c|}{$\mathrm{V}$}} \\
\hline & $\mathrm{H}_{2} \mathrm{O}$ & & & & & $\mathrm{cmc}$ & $\mathrm{dm}^{3}$ & & & & \\
\hline LVA distrófico & 5,20 & 0,80 & 49 & 0,30 & 0,00 & 0,00 & 1,82 & 0,43 & 2,25 & 19 & 0 \\
\hline
\end{tabular}

pH em água - Relação 1:2,5

$\mathrm{P}$ e K - Extrator Mehlich 1.

CTC (T) - Capacidade de troca catiônica $(\mathrm{pH} 7,0)$.

$\mathrm{Ca}^{2+}, \mathrm{Mg}^{2+} \mathrm{e} \mathrm{Al}^{3+}$ - Extrator $\mathrm{KCl} 1 \mathrm{~mol} / \mathrm{L}$

$\mathrm{H}+\mathrm{Al}-$ Extrator Acetato de Cálcio 0,5 mol/L - pH 7,0
SB - Soma de bases trocáveis

$\mathrm{V}$ - Índice de Saturação por bases

MO - C. Org x 1,724-Método Walkley-Black

$\mathrm{m}$ - Saturação por alumínio

Tabela 2 - Apresentação dos tratamentos com doses aplicadas dos nutrientes em cada um deles.

Table 2 - Treatments with respective applied doses of nutrients.

\begin{tabular}{cccc}
\hline Tratamentos & Unidades & Tratamentos & Unidades \\
\hline DR & $\mathrm{mg} / \mathrm{dm}^{3} / \mathrm{cmol}_{\mathrm{c}} / \mathrm{dm}^{3}$ & $\mathrm{~K}=200 * *$ & $\mathrm{mg} / \mathrm{dm}^{3}$ \\
Solo sem correção & - & $\mathrm{Ca}=0,8 * *$ & $\mathrm{cmol} / \mathrm{dm}^{3}$ \\
$\mathrm{~N}=50 * *$ & $\mathrm{mg} / \mathrm{dm}^{3}$ & $\mathrm{Ca}=1,2 * *$ & $\mathrm{cmol} / \mathrm{dm}^{3}$ \\
$\mathrm{~N}=150 * *$ & $\mathrm{mg} / \mathrm{dm}^{3}$ & $\mathrm{Ca}=1,4 * *$ & $\mathrm{Mg}=0,2 * *$ \\
$\mathrm{~N}=200 * *$ & $\mathrm{mg} / \mathrm{dm}^{3}$ & $\mathrm{Mg}=0,6 * *$ & $\mathrm{c} / \mathrm{dm}^{3}$ \\
$\mathrm{P}=150 * *$ & $\mathrm{mg} / \mathrm{dm}^{3}$ & $\mathrm{Mg}=0,8 * *$ & $\mathrm{cmol}_{\mathrm{c}} / \mathrm{dm}^{3}$ \\
$\mathrm{P}=450 * *$ & $\mathrm{mg} / \mathrm{dm}^{3}$ & $\mathrm{~S}=20 * *$ & $\mathrm{cmol}_{\mathrm{c}} / \mathrm{dm}^{3}$ \\
$\mathrm{P}=600 * *$ & $\mathrm{mg} / \mathrm{dm}^{3}$ & $\mathrm{~S}=60 * *$ & $\mathrm{cmol}_{\mathrm{c}} / \mathrm{dm}^{3}$ \\
$\mathrm{~K}=50 * *$ & $\mathrm{mg} / \mathrm{dm}^{3}$ & $\mathrm{~S}=80 * *$ & $\mathrm{mg} / \mathrm{dm}^{3}$ \\
$\mathrm{~K}=150 * *$ & $\mathrm{mg} / \mathrm{dm}^{3}$ & & $\mathrm{mg} / \mathrm{dm}^{3}$ \\
\hline
\end{tabular}

* Doses de referência (DR): $\mathrm{N}=100 \mathrm{mg} / \mathrm{dm}^{3} ; \mathrm{P}=300 \mathrm{mg} / \mathrm{dm}^{3} ; \mathrm{K}=100 \mathrm{mg} / \mathrm{dm}^{3} ; \mathrm{Ca}=1 \mathrm{cmol} / \mathrm{dm}^{3} ; \mathrm{Mg}=0,4 \mathrm{cmol}_{\mathrm{c}} / \mathrm{dm}^{3}$; e $\mathrm{S}=40 \mathrm{mg} / \mathrm{dm}^{3}$.

** Em cada tratamento, apenas o nutriente indicado tem a dose variando, permanecendo as doses dos demais nutrientes iguais à dose de referência.

Antes da semeadura foi aplicada uma solução de micronutrientes com boro (B) $\left(0,81 \mathrm{mg} / \mathrm{dm}^{3}\right.$ de $\left.\mathrm{H}_{3} \mathrm{BO}_{3}\right)$, cobre $(\mathrm{Cu})\left(1,33 \mathrm{mg} / \mathrm{dm}^{3}\right.$ de $\left.\mathrm{CuSO}_{4} .5 \mathrm{H}_{2} \mathrm{O}\right)$, molibdênio (Mo) $\left(0,15 \mathrm{mg} / \mathrm{dm}^{3} \mathrm{de}\left(\mathrm{NH}_{4}\right)_{6} \mathrm{Mo}_{7} \mathrm{O}_{24} \cdot 4 \mathrm{H}_{2} \mathrm{O}\right)$, manganês (Mn) $\left(3,66 \mathrm{mg} / \mathrm{dm}^{3} \mathrm{de} \mathrm{MnCl}_{2} \cdot \mathrm{H}_{2} \mathrm{O}\right)$ e zinco $(\mathrm{Zn})(4,0$ $\mathrm{mg} / \mathrm{dm}^{3} \mathrm{de} \mathrm{ZnSO}_{4} \cdot 7 \mathrm{H}_{2} \mathrm{O}$ ), conforme indicado por Alvarez V. (1974).

A unidade experimental foi composta por um vaso de polietileno rígido, com capacidade para $2,1 \mathrm{dm}^{3} \mathrm{de}$ solo. Para produção das mudas, fez-se a semeadura direta, colocando 10 sementes por vaso, e realizando o primeiro raleio aos 15 dias após a emergência das mudas e deixando duas mudas por vaso. O segundo raleio foi feito aos 30 dias, deixando-se apenas uma muda por vaso. $\mathrm{O}$ teor de umidade do solo foi mantido próximo a $60 \%$ da capacidade de campo, através de um monitoramento diário.

\subsection{Análise dos dados}

O experimento foi encerrado120 dias após a semeadura. Fez-se a medição de altura das mudas com régua graduada em centímetros e do diâmetro do coleto com paquímetro com precisão de 0,01 mm. As plantas foram colhidas, subdivididas em raiz, caule e folhas, lavadas em água destilada e secas em estufa com circulação forçada de ar a $60^{\circ} \mathrm{C}$ até atingir peso constante. Depois de seco, o material foi pesado em balança analítica com precisão de $0,01 \mathrm{~g}$, para determinar o peso de matéria seca de raiz (MSR), o peso de matéria seca do caule (MSC) e o peso de matéria seca de folhas (MSF).

Calcularam o peso de matéria seca de parte aérea (MSPA), o peso de matéria seca total (MST) e, ainda, os índices de qualidade de mudas: altura de parte aérea por diâmetro do coleto (H/D), altura de parte aérea 
por peso de matéria seca de parte aérea (H/MSPA), e peso de matéria seca de parte aérea por peso de matéria seca de raiz (MSPA/MSR), bem como o índice de qualidade de Dickson (IQD), de acordo com a fórmula (DICKSON et al., 1960):

$$
\mathrm{IQD}=\frac{\operatorname{MST}(g)}{\mathrm{H}(\mathrm{cm}) / \mathrm{D}(\mathrm{mm})+\operatorname{MSPA}(g) / \operatorname{MSR}(g)}
$$

Os dados obtidos foram interpretados estatisticamente através da análise dos contrastes ortogonais para verificar a existência de efeito significativo dos tratamentos aplicados. Com o software SAEG (Sistema de Análises Estatísticas e Genética), procedeu-se a análises de variância individuais para estimar o erro experimental e de regressão, escolhendo o modelo de melhor ajuste aos dados com base no coeficiente de determinação $\left(\mathrm{R}^{2}\right)$ (EUCLYDES, 1997). $\mathrm{O}$ ajuste das equações de regressão foi feito, testandose os respectivos coeficientes pelo teste "t", de Student, com base no quadrado médio do resíduo da ANOVA. Dessa forma, diante de dois ou mais modelos com coeficientes significativos, optou-se pelo de maior $\mathrm{R}^{2}$. Com essas equações, procurou-se determinar doses críticas dos macronutrientes, para obtenção de $90 \%$ dos valores máximos estimados das variáveis estudadas.

\section{RESULTADOS}

As plantas de Senna macranthera demonstraram, pela análise dos contrastes ortogonais, resposta significativa de todas as variáveis estudadas à aplicação de doses crescentes dos macronutrientes.

\subsubsection{Altura $(\mathbf{H})$}

Para os tratamentos N, K, Ca e S não foram encontrados modelos matemáticos que se ajustassem adequadamente às respostas observadas e significativas de Senna macranthera. Já por ocasião da aplicação de doses de fósforo ao substrato-se, observou-se, resposta linear crescente do crescimento em altura das plantas. Devido à resposta apresentada, a maior altura tende a ocorrer para aplicação de uma dose de $\mathrm{P}$ superior à maior dose aplicada $\left(600 \mathrm{mg} / \mathrm{dm}^{3}\right)$, impedindo, assim, a determinação de uma dose crítica do nutriente. Já na aplicação de doses de magnésio ao substrato as plantas de Senna macranthera apresentaram resposta linear decrescente de crescimento em altura com o aumento da dose aplicada do nutriente (Figura 1).
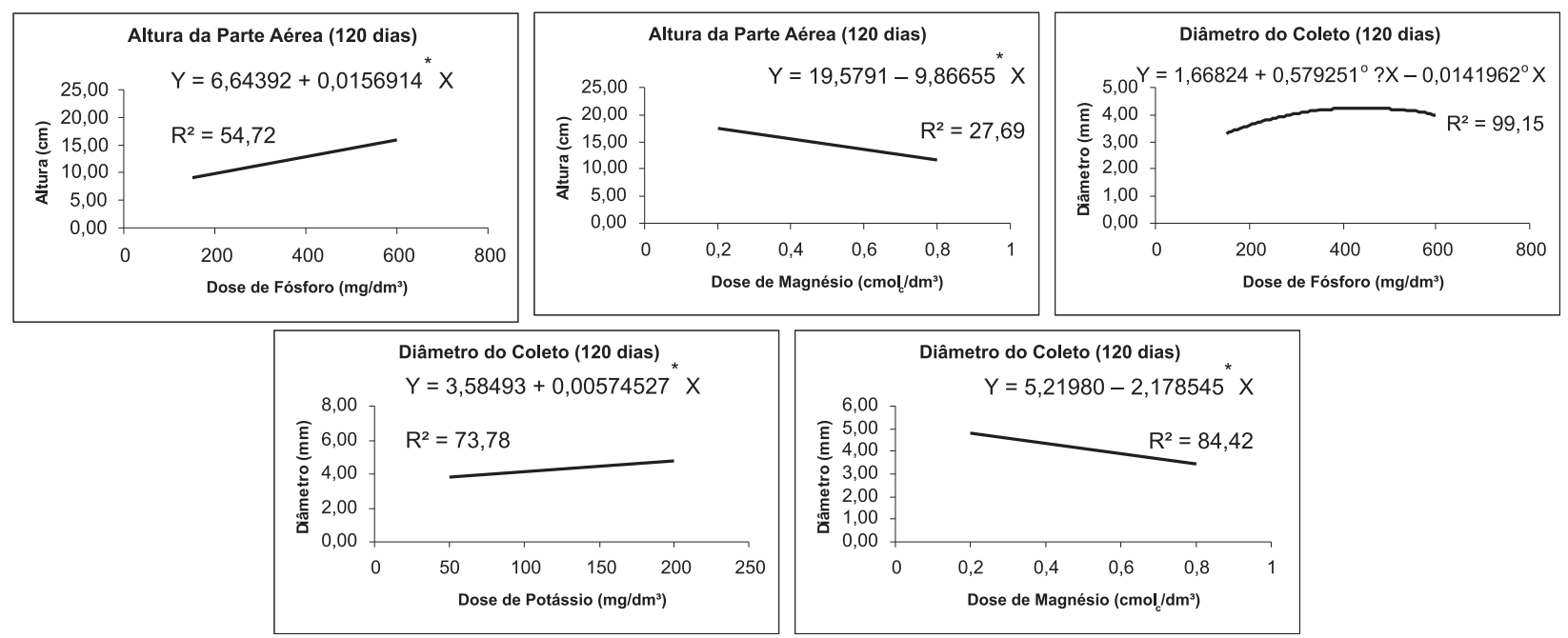

Figura 1 - Altura da parte aérea e diâmetro do coleto das plantas de Senna macranthera (fedegoso) em resposta a doses crescentes de fósforo e magnésio, em Latossolo Vermelho-Amarelo distrófico, 120 dias após a semeadura.

Figure 1 - Aerial part height and root collar diameter of Senna macranthera (fedegoso) plants in response to increasing doses of phosphorus and magnesium, in dystrophic red yellow latosol, 120 days after sowing.

R. Árvore, Viçosa-MG, v.34, n.1, p.13-24, 2010 


\subsubsection{Diâmetro (D)}

Nos tratamentos N, Ca e S não foram encontrados modelos matemáticos que se ajustassem adequadamente às respostas observadas de crescimento em diâmetro das mudas de Senna macranthera. Já no tratamento P foram verificadas respostas do crescimento em diâmetro do coleto das mudas explicadas por um modelo raiz quadrada, com seu máximo valor $(4,24 \mathrm{~mm})$ sido estimado para a dose calculada de $416,23 \mathrm{mg} / \mathrm{dm}^{3}$ e para a dose crítica em $223,09 \mathrm{mg} / \mathrm{dm}^{3}$.

Para a aplicação de doses de potássio ao substrato, verificou-se resposta linear crescente do crescimento em diâmetro das mudas, enquanto no tratamento $\mathrm{Mg}$ se observou resposta linear decrescente à aplicação de doses desse nutriente.

\subsubsection{Produção de matéria seca de parte área (MSPA), raiz (MSR) e total (MST)}

No caso dos tratamentos N, Ca e K aplicados sobre as mudas de Senna macranthera, não foram encontrados modelos matemáticos que se ajustassem adequadamente às respostas observadas.

No tratamento $\mathrm{P}$, as plantas apresentaram resposta quadrática negativa de produção de MSPA, MSR e MST à aplicação de doses do nutriente. No caso do primeiro, o máximo valor de MSPA $(3,11 \mathrm{~g})$ foi estimado para a dose calculada de $434,99 \mathrm{mg} / \mathrm{dm}^{3}$ e dose crítica estimada em $325,63 \mathrm{mg} / \mathrm{dm}^{3}$. Para MSR a máxima produção $(1,58 \mathrm{~g})$ foi estimada para a dose calculada de 396,09 $\mathrm{mg} / \mathrm{dm}^{3}$ e para dose crítica de $272,43 \mathrm{mg} / \mathrm{dm}^{3}$, enquanto para MST a máxima produção $(4,67 \mathrm{~g})$ foi estimada para a dose calculada de $423,94 \mathrm{mg} / \mathrm{dm}^{3}$ e para a dose crítica em $310,47 \mathrm{mg} / \mathrm{dm}^{3}$ (Figura 2).
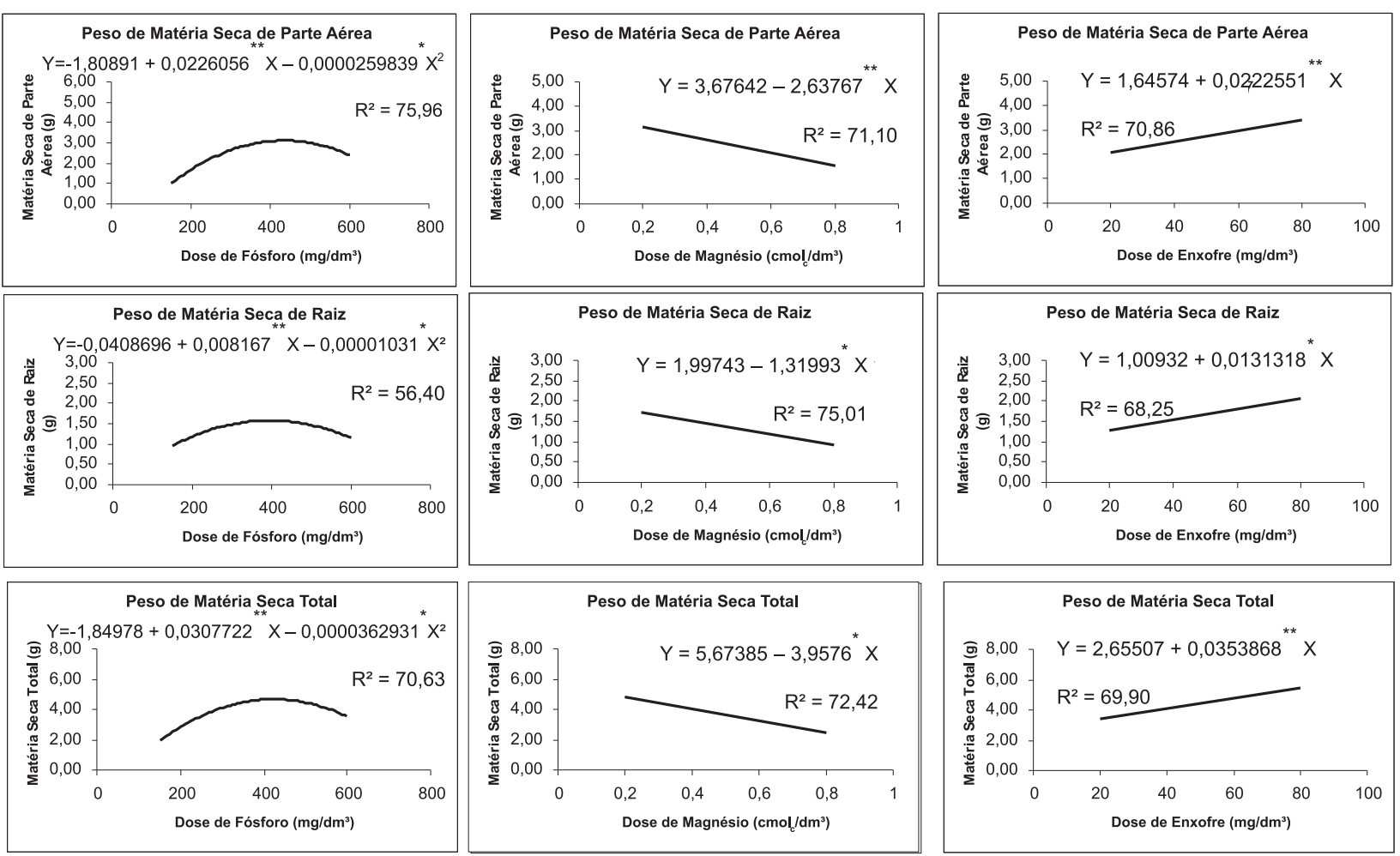

Figura 2 - Produção de matéria seca da parte aérea, raiz e total das plantas de Senna macranthera (fedegoso) em resposta à aplicação de doses crescentes de fósforo, magnésio e enxofre, em Latossolo Vermelho-Amarelo distrófico, 120 dias após a semeadura.

Figure 2 - Dry matter of the aerial part, root and total production of Senna macranthera (fedegoso) in response to increasing doses of phosphorus, magnesium and sulfur, in a dystrophic red yellow latosol, 120 days after sowing. 
No tratamento Mg observou-se reposta linear decrescente da MSPA, MSR e MST de Senna macranthera, com diminuição no valor das variáveis em resposta ao aumento da dose do nutriente aplicada, e indicando que o maior valor das variáveis ocorrerá em uma dose inferior à menor dose do nutriente testada $\left(0,2 \mathrm{cmol}_{\mathrm{c}} / \mathrm{dm}^{3}\right)$.

As plantas de Senna macranthera apresentaram, também, resposta significativa à aplicação de doses de enxofre para produção de matéria seca de parte aérea, raiz e total, observando-se resposta linear crescente dessas variáveis ao aumento da dose aplicada do nutriente.

\subsection{4. Índices de qualidade de mudas}

As plantas de Senna macranthera apresentaram respostas distintas entre si para H/D no caso dos tratamentos $\mathrm{NeP}$ (Figura 3). No primeiro ocorreu diminuição linear dos valores observados para essa variável com o aumento da dose de $\mathrm{N}$ aplicada ao substrato. No
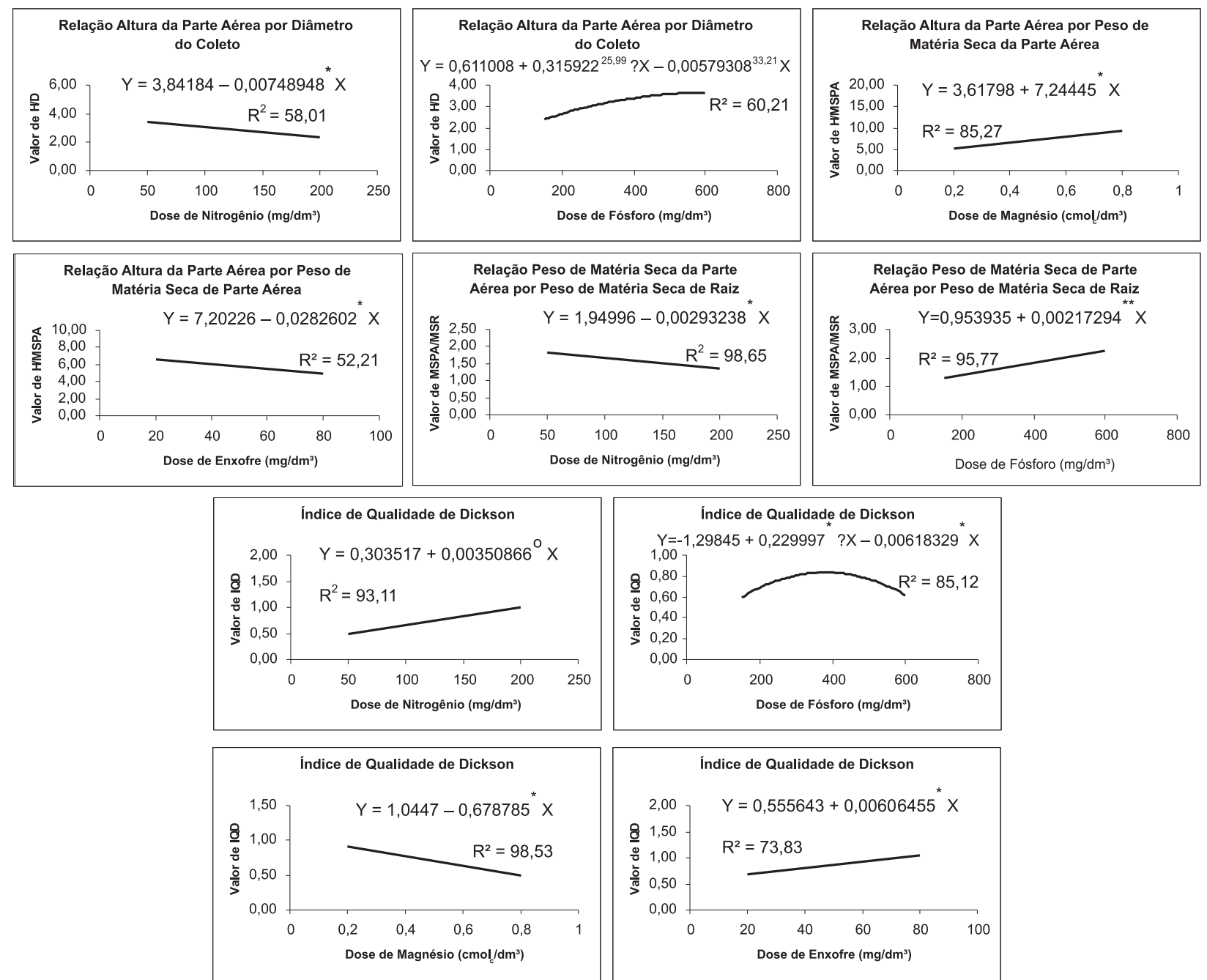

Figura 3 - Dados da altura da parte aérea por diâmetro do coleto (H/D); altura da parte aérea pelo peso de matéria seca de parte aérea (H/MSPA); peso de matéria seca de parte aérea pelo peso de matéria seca de raiz (MSPA/MSR); e índice de qualidade de Dickson (IQD) das plantas de Senna macranthera (fedegoso) em resposta a doses crescentes de nitrogênio e fósforo em Latossolo Vermelho-Amarelo distrófico, 120 dias após a semeadura.

Figure 3 - Aerial part height/root collar diameter (H/D); aerial part height/aerial part dry matter ratio (H/MSPA); aerial part dry matter/root dry matter ratio (MSPA/MSR) and Dickson quality index (IQD) of Senna macranthera (fedegoso) in response to increasing doses of nitrogen and phosphorus in a dystrophic red yellow latosol, 120 days after sowing.

R. Árvore, Viçosa-MG, v.34, n.1, p.13-24, 2010 
segundo, a resposta foi interpretada por um modelo raiz quadrada, com o maior valor de H/D $(3,70)$ tendo sido estimado para a dose calculada de 743,50 $\mathrm{mg} / \mathrm{dm}^{3}$ e para a dose crítica de $\mathrm{P}$ determinada em $371,70 \mathrm{mg} / \mathrm{dm}^{3}$.

Nos demais tratamentos aplicados, K, Ca, Mg e $\mathrm{S}$ não foi encontrado um modelo matemático que se ajustasse adequadamente às respostas observadas de H/D. Neste trabalho, verificou-se que Senna macranthera apresentou valores de H/D variando entre 2,02 e 4,24, com maior concentração dos dados observados entre 2,3 e 3,7 .

Para H/MSPA, apenas nos tratamentos Mg e S foram encontrados modelos matemáticos que se ajustassem adequadamente às respostas observadas. No tratamento $\mathrm{Mg}$, observou-se aumento linear dos valores de H/MSPA com o incremento da dose aplicada do nutriente. Esse resultado se opõe ao observado no tratamento $\mathrm{S}$, em que os valores de H/MSPA diminuíram linearmente em resposta ao aumento da dose aplicada do nutriente. Neste estudo, verificaram-se valores de H/MSPA variando entre 4,87 e 18,82, com a concentração dos dados próximos a 5,0.

Às respostas observadas de MSPA/MSR nos tratamentos K, Ca, Mg e S não foram encontrados modelos matemáticos que se ajustassem adequadamente. Nos tratamentos N e P, observaram-se respostas de MSPA/ MSR opostas entre si. Para N, verificou-se diminuição linear dos valores observados na relação com o aumento da dose do nutriente aplicada ao substrato. Já o aumento da dose de $\mathrm{P}$ adicionada ao substrato acarretou aumento linear dos valores da relação. Verificou-se ainda, neste estudo, que MSPA/MSR variou entre 0,83 e 2,18 .

Neste trabalho, verificou-se efeito significativo de todos os tratamentos sobre o IQD de Senna macranthera. Entretanto, nos tratamentos $\mathrm{K}$ e Ca não foram encontrados modelos matemáticos que se ajustassem adequadamente às respostas. Observaram-se, ainda, respostas lineares crescentes aos tratamentos $\mathrm{N}$ e $\mathrm{S}$, enquanto no caso do tratamento $\mathrm{Mg}$ foi verificada resposta linear decrescente da variável com o aumento da dose do nutriente aplicado. No tratamento $P$, verificou-se resposta das mudas de Senna macranthera, explicada por um modelo raiz quadrada, em que o máximo valor de IQD $(0,84)$ foi estimado para a dose calculada de $345,90 \mathrm{mg} / \mathrm{dm}^{3}$, sendo a dose crítica do nutriente estimada em 222,36 $\mathrm{mg} / \mathrm{dm}^{3}$. Neste estudo, verificaram-se valores de IQD variando entre 0,20 e 1,05 .

\section{DISCUSSÃO}

\subsection{Efeito da aplicação de macronutrientes}

\subsubsection{Nitrogênio}

De acordo com Gonçalves et al. (2008), efeitos no crescimento de plantas em virtude da aplicação de $\mathrm{N}$ são esperados em razão das diversas funções exercidas pelo nutriente dentro destas. Tal fato se confirmou para as mudas de fedegoso neste estudo, em que foi observada a resposta significativa das mudas aos tratamentos aplicados.

Venturin et al. (1999) também verificaram respostas significativas à adição de nutrientes ao substrato, trabalhando com mudas de Peltophorum dubium, observando que o crescimento em altura e diâmetro, bem como a produção de matéria seca de parte aérea e raiz das plantas, diminuiu ao se omitirem os macronutrientes do meio de crescimento. Em contrapartida, Duboc et al. (1996) demonstraram haver espécies com baixo requerimento nutricional para os macronutrientes, como Hymenaea courbaril, para a qual verificaram que a omissão de $\mathrm{N}$ não teve efeito significativo sobre as já referidas variáveis.

O comportamento verificado para MSPA/MSR e para o IQD das plantas de fedegoso, neste estudo foi similar aos observados em mudas de Jacaré por Marques et al. (2009), que verificaram que os melhores valores dessas relações ocorreram nas doses de $59,90 \mathrm{mg} / \mathrm{dm}^{3}$ e $190 \mathrm{mg} / \mathrm{dm}^{3}$, respectivamente, valores próximos da menor e maior dose aplicada, respectivamente, neste trabalho.

Os resultados aqui verificados nas mudas de fedegoso, assim como em outros trabalhos com diferentes espécies florestais encontrados na literatura, evidenciaram que os melhores resultados na produção de mudas, considerados os resultados das diferentes variáveis analisadas, ocorrem de forma geral para a aplicação de doses de nitrogênio superiores a $170 \mathrm{mg} / \mathrm{dm}^{3}$ (MARQUES et al., 2006; MARQUES et al., 2009; GONÇALVES et al., 2008).

R. Árvore, Viçosa-MG, v.34, n.1, p.13-24, 2010 


\subsubsection{Fósforo}

O fósforo é um dos nutrientes mais utilizados na fertilização mineral de substratos. Tal fato se verificou devido à sua carência na maioria dos solos tropicais e constitui importante fator de limitação para o crescimento de espécies florestais exóticas e nativas (FERNANDES et al., 2007; SOUZA et al., 2006). Tendo em vista o fato exposto, são comuns na literatura os relatos do efeito significativo da aplicação do nutriente, fato esse que se confirmou, também, nas mudas de fedegoso nesse estudo, em todas as variáveis analisadas.

Este trabalho indicou, entre as variáveis, que as doses críticas do nutriente variaram entre 223,09 e 371,70 $\mathrm{mg} / \mathrm{dm}^{3}$, sendo esses valores maiores do que os observados por Gonçalves et al. (2008) em mudas de angico-vermelho e que recomendaram doses que variaram de 150 a 250 $\mathrm{mg} / \mathrm{dm}^{3}$, conforme o solo utilizado. Tal fato demonstra que o fedegoso se mostrou mais exigente que a espécie anterior nas condições experimentais adotadas. Trabalhando com Cordia goeldiana, Fernandes et al. (2002) verificaram que a adubação fosfatada promoveu aumento linear no crescimento em altura das mudas, à semelhança do constatado no fedegoso. Mas, tendo em vista o valor da maior dose testada pelos pesquisadores $\left(450 \mathrm{mg} / \mathrm{dm}^{3}\right)$, pode-se inferir que também aqui o fedegoso se mostrou mais exigente nutricionalmente de $\mathrm{P}$.

Lima et al. (2008) verificaram comportamento de Euterpe edulis que indica respostas lineares positivas nas diferentes variáveis até a dosagem de $540 \mathrm{mg} / \mathrm{dm}^{3}$. Tais respostas demonstram que essa espécie se apresenta consideravelmente mais exigente de $\mathrm{P}$ que o fedegoso, nas condições experimentais utilizadas pelos pesquisadores. Da mesma forma, em mudas de Myracrodruon urundeuva, Schinus terebinthifolius, Piptadenia gonoacantha, Mimosa caesalpinifolia e Sesbania virgata, Resende et al. (1999) observaram efeito significativo da aplicação de fósforo ao substrato sobre o crescimento em diâmetro das mudas, determinando doses críticas de 452,62; 377,35; 480,96; 800,00 ; e $215,10 \mathrm{mg} / \mathrm{dm}^{3}$, respectivamente, e, em sua maioria, bem maiores do que as verificadas em Senna macranthera neste estudo.

\subsubsection{Potássio}

Respostas significativas como a verificada no fedegoso à aplicação de K são encontradas na literatura, sendo, entretanto, os resultados obtidos com as

R. Árvore, Viçosa-MG, v.34, n.1, p.13-24, 2010 diferentes espécies florestais bastante diferenciados. Venturin et al. (1999) observaram em Peltophorum dubium que, na omissão de K, houve menor crescimento em altura sem no entanto, diferir do tratamento completo. Já Reis et al. (1997) verificaram em Dalbergia nigra, resposta quadrática à aplicação de potássio ao substrato, tendo determinado para a espécie uma dose crítica do nutriente de $30 \mathrm{mg} / \mathrm{dm}^{3}$.

Gonçalves et al. (2008) observaram em angicovermelho que o potássio, à semelhança do que se verifica em outros trabalhos na literatura, foi o nutriente que provocou menores efeitos significativos nas variáveis estudadas, o que, segundo esses autores, indica que nessa espécie a necessidade do nutriente pela planta pode ter sido suprida pelos teores originalmente presentes no solo.

Neste estudo, tendo em vista as respostas observadas em todas as variáveis utilizadas na aplicação do nutriente, em especial no diâmetro do coleto, onde a análise de regressão conseguiu explicar os resultados, verificou-se uma resposta indicando ser o fedegoso mais exigente nutricionalmente em potássio que as espécies anteriores.

\subsubsection{Cálcio e magnésio}

A resposta das plantas à aplicação de cálcio e de magnésio ao substrato pode estar muito ligada ao teor dos nutrientes pré-existente no solo, no que concordaram Neves et al. (1982), que afirmaram que o uso de calagem para a produção de mudas seria necessária quando os níveis de cálcio e magnésio do solo fossem inferiores a 0,2 e $0,06 \mathrm{cmol}_{\mathrm{c}} / \mathrm{dm}^{3}$, respectivamente.

No caso do cálcio, verificou-se que o solo apresentou, neste estudo, valor superior ao relatado pelos autores anteriores, e ainda assim o fedegoso apresentou resposta significativa à aplicação do nutriente ao substrato, indicando ser essa espécie mais exigente no nutriente. O mesmo tipo de resposta foi observado na adição de magnésio ao substrato, ressaltando-se aqui, entretanto, que o solo apresentava um nível original de $0,00 \mathrm{cmol}_{\mathrm{c}} / \mathrm{dm}^{3}$, justificando também, por isso resposta observada ao nutriente em questão.

A resposta à adição de cálcio e magnésio ao substrato através da calagem sobre determinadas características de crescimento das mudas vai depender das características da espécie e, especialmente, da sua 
tolerância à acidez do solo (VALE et al., 1996). Braga et al. (1995) observaram em Aspidosperma polyneuron que a omissão de cálcio não afetou a produção de matéria seca radicular das plantas, demonstrando ser essa espécie menos exigente do nutriente do que o fedegoso. Da mesma forma, contrariando o observado na espécie aqui estudada, Duboc et al. (1996) observaram em Hymenaea courbaril que a omissão de magnésio não afetou significativamente a produção de matéria seca de parte aérea e de raiz das plantas, tendo, sob omissão, ocorrido aumento nos valores dessas variáveis.

Gonçalves et al. (2008) verificaram que plantas de angico-vermelho apresentou baixa resposta à adição de cálcio ao substrato e, no caso do magnésio, diferença no crescimento só foi detectada em algumas das variáveis estudadas quando as plantas foram cultivadas em Latossolo Vermelho-Amarelo álico. Em contrapartida, Souza et al . (2008) verificaram em Machaerium nictitans que, sem a adição de mistura corretiva ao substrato, as variáveis estudadas apresentaram as menores médias, demonstrando o efeito positivo da adição de $\mathrm{Ca}$ e $\mathrm{Mg}$ ao substrato, assim como verificado no fedegoso, neste estudo.

Os resultados apresentados pelas plantas de fedegoso à aplicação de $\mathrm{Mg}$ no substrato, neste estudo demonstraram, de forma geral, que os melhores valores das diferentes variáveis estudadas vão estar próximos da menor dose aplicada do nutriente ao substrato utilizado $\left(0,2 \mathrm{cmol}_{\mathrm{c}} / \mathrm{dm}^{3}\right)$. Essa dose coincide com a dose recomendada para angico-vermelho por Gonçalves et al. (2008), indicando que essas espécies apresentaram exigências nutricionais em magnésio semelhantes.

\subsubsection{Enxofre}

A importância da adubação com enxofre para o crescimento em diâmetro das plantas é ressaltada por Braga et al. (1995), que observaram que a omissão de enxofre no substrato comprometeu o crescimento em diâmetro de Tibouchina granulosa e Platycyamus regnellii conduzidas por 100 dias. Neste trabalho, as plantas de fedegoso também se mostraram significativamente responsivas à adição do nutriente ao substrato, concordando com o verificado por esses autores em seus trabalhos.

Resposta significativa de produção de matéria seca de espécies florestais nativas à adubação com enxofre também foi verificada por Fernández et al. (1996) em Mimosa tenuiflora, os quais determinaram uma dose crítica do nutriente de $14,0 \mathrm{mg} / \mathrm{dm}^{3}$ para a produção de matéria seca de parte aérea. Também Gonçalves et al. (2008), trabalhando com mudas dessa espécie, verificaram resposta significativa a adição de enxofre ao substrato tendo recomendado doses que variaram entre 20 e $80 \mathrm{mg} / \mathrm{dm}^{3}$ conforme o solo utilizado no trabalho.

A análise das respostas apresentadas pelas plantas de fedegoso neste estudo demonstrou que os melhores resultados alcançados na maior parte das variáveis estudadas ocorrem, de forma geral, com a maior dose aplicada do nutriente neste estudo $\left(80 \mathrm{mg} / \mathrm{dm}^{3}\right)$, demonstrando ser a espécie anterior bastante exigente no nutriente em questão.

\subsection{Resposta das diferentes variáveis estudadas}

\subsubsection{Parâmetros morfológicos}

De acordo com vários autores, mudas de espécies arbóreas estão aptas para o plantio no campo quando a altura da parte aérea estiver entre 15 e $30 \mathrm{~cm}$ (PAIVA e GOMES, 2000). Neste estudo, aos 120 dias de idade apenas em alguns dos tratamentos aplicados os valores médios de altura se encontravam entre os supramencionados, podendo-se afirmar, assim, que as plantas de fedegoso, por esse critério, não estariam aptas para plantio no campo.

Já quanto ao diâmetro do coleto Carneiro (1995) afirmou que mudas com maiores diâmetros do coleto apresentam melhor equilíbrio do crescimento da parte aérea. Vários pesquisadores indicaram como ideais para as espécies florestais diferentes valores desse parâmetro morfológico, mas sempre superiores a 6,4 mm, obtido em Liquidambar styraciflua por Belanger e Mcalpine (1975), citados por GOMES (2001). Neste trabalho, verificaram-se em fedegoso valores de diâmetro aos 120 dias de idade sempre superiores a 3,0 $\mathrm{mm}$ e, de forma geral, próximos de 4,0 mm, estando por isso perto dos estipulados para a espécie anterior.

Quanto à produção de matéria seca das plantas, observou-se que essa é interessante para que apresentem maior vigor e capacidade fotossintética, levando ao melhor desenvolvimento das plantas; pode-se inferir que o desejável para essa variável se encontra no seu máximo. Neste trabalho, verificaram-se respostas variadas, indicando ser o fedegoso significativamente exigente quanto aos macronutrientes para seu bom desenvolvimento na fase juvenil.

R. Árvore, Viçosa-MG, v.34, n.1, p.13-24, 2010 


\subsection{2. Índices de qualidade de mudas}

O valor de H/D é um importante índice e, quanto menor seu valor, maior a capacidade de sobrevivência das mudas e de estabelecimento após o plantio definitivo (CARNEIRO, 1983). Neste trabalho, verificou-se ainda que, em Senna macranthera, os valores de H/D observados estão próximos dos verificados por Cruz et al. (2004) em Tabebuia impetiginosa, que observaram valores médios de $\mathrm{H} / \mathrm{D}$ variando entre 2,93 e 3,32.

De acordo com Gomes (2001), o quociente obtido pela divisão da altura da parte aérea pelo peso de matéria seca da parte aérea não tem uso frequente para avaliação do padrão de qualidade de mudas, podendo, entretanto, ser de grande valia,e, quanto menor o valor de H/MSPA, mais lenhificada a muda e maior deverá ser a sua capacidade de sobrevivência no campo. Neste trabalho, verificaram-se valores de H/MSPA em Senna macranthera bem acima dos encontrados por Cruz et al. (2004) que encontraram valores de H/MSPA variando entre 1,32 e 1,74 em Tabebuia impetiginosa.

A relação entre o peso de matéria seca da parte aérea e o de raiz é considerado um eficiente índice, além de seguro, para expressar o padrão de qualidade das mudas (LIMSTRON, 1963, citado por GOMES, 2001). Brissette (1984) mencionou que, num encontro de pesquisadores, ficou estabelecido como 2,0 a melhor relação entre o peso de matéria seca de parte aérea e o de raiz de uma mesma planta. Em Senna macranthera, verificaram-se, neste estudo, valores de MSPA/MSR próximos dos observados por Cruz et al. (2004) em Tabebuia impetiginosa, os quais obtiveram valores variando entre 1,18 e 1,65 .

Quanto ao IQD, o referido índice é uma fórmula balanceada que inclui as relações entre os parâmetros morfológicos. Desenvolvida por Dickson et al. (1960) em mudas de Picea glauca e Pinus monficola, eles ressaltaram que, quanto maior o valor encontrado para esse índice, melhor será o padrão de qualidade das mudas (GOMES 2001). Neste estudo, verificaram-se em Senna macranthera valores de IQD observados, bem inferiores aos encontrados em Tabebuia impetiginosa, cujos valores médios variaram de 6,21 a 7,25 (CRUZ et al., 2004).

\section{CONCLUSÃO}

As plantas de fedegoso mostraram-se responsivas à adição de macronutrientes ao substrato em todas as variáveis analisadas.
Para a adição de nitrogênio, potássio e enxofre, apesar de significativas as respostas observadas nos respectivos tratamentos, não foi possível determinar o valor de dose crítica desses nutrientes.

Em resposta à adição de doses de $\mathrm{P}$ ao substrato, observou-se ser a espécie estudada bastante exigente desse nutriente, comparativamente com outras espécies, tendo-se verificado valores de doses críticas entre 222,36 $\mathrm{mg} / \mathrm{dm}^{3}$ e $335,00 \mathrm{mg} / \mathrm{dm}^{3}$.

Para a adição de cálcio e magnésio ao substrato, verificou-se resposta significativa da adição desses nutrientes ao substrato, explicada principalmente pelo baixo nível encontrado deles no solo utilizado.

Ficou evidente neste trabalho a necessidade de maior diferenciação entre as doses aplicadas de cada um dos nutrientes, bem como a instalação de uma testemunha para cada tratamento. Observou-se ser interessante ainda, devido ao crescimento mais lento da espécie comparativamente a outras já estudadas, aumentar o tempo experimental.

\section{AGRADECIMENTOS}

A Deus, primeiramente; à minha esposa Natane, meus amigos Catarina e Felipe; à UFV, pelo oferecimento do Curso de Mestrado em Ciência Florestal; ao CNPq, pela bolsa concedida; ao projeto PRODETAB 130-02/ 01, pelo apoio financeiro; aos funcionários do Viveiro do DEF; e ao Prof. Haroldo, pela orientação paciente dada durante todo o trabalho.

\section{REFERENCIAS}

ALVAREZ V., V. H. Equilíbrio de formas disponíveis de fósforo e enxofre em dois Latossolos de Minas Gerais. 1974. 125f. Dissertação (Mestrado em Solos e Nutrição de Plantas) - Universidade Federal de Viçosa, Viçosa, MG, 1974.

BRAGA, F. A. et al. Exigências nutricionais de quatro espécies florestais. Revista Árvore, v.19, n.1, p.18-31, 1995.

BRISSETTE, J. C. Summary of discussions about seedling quality. In: SOUTHERN NURSERY CONFERENCES, 1984, Alexandria.

Proceedings... New Orleans: USDA. Forest Service. Southern Forest Experiment Station, 1984. p.127-128. 
CARneiro, J. G. A. Produção e controle de qualidade de mudas florestais. Curitiba: UFPR/FUPEF, 1995. 451p.

CARNEIRO, J. G. A. Variações na metodologia de produção de mudas florestais afetam os parâmetros morfofisiológicos que indicam a sua qualidade. Série Técnica FUPEF, n.12, p.1-40, 1983.

CARVALHO, M. M. Ações visando recuperação de áreas de pastagens degradadas. In: ENCONTRO PARA CONSERVAÇÃO DA NATUREZA, 1., 1997,Viçosa. Ánais... Viçosa, MG: CMCN/DEF/UFV, 1997. p.202-206.

CRUZ, C. A. F. et al. Efeito de diferentes níveis de saturação por bases no desenvolvimento e qualidade de mudas de ipê-roxo (Tabebuia impetiginosa (Mart.) Standley). Revista Scientia Forestalis, n.66, p.100-107, 2004.

DICKSON, A.; LEAF, A. L.; HOSNER, J. F. Quality appraisal of white spruce and white pine seedling stock in nurseries. Forestry Chronicle, v.36, n.1, p.10-13, 1960.

DUBOC, E. et al. Nutrição do jatobá (Hymenaea courbaril L. var. Stilbocarpa (Hayne) Lee et Lang.) Revista Cerne, v.2, n.1, p.31-47, 1996.

DNM - DEPARTAMENTO NACIONAL DE METEOROLOGIA. Normais climatológicas (1961-1990). Brasília: 1992. 84p.

EUCLYDES, R. F. Manual de utilização do programa SAEG (Sistema para análises estatísticas e genéticas) Viçosa, MG: Universidade Federal de Viçosa, 1997. 59p.

FERNANDES, L. A. et al. Crescimento inicial, níveis críticos de fósforo e frações fosfatadas em espécies florestais. Pesquisa Agropecuária Brasileira, v.35, n.6, p.1191-1198, 2000.

FERNANDES, A. R. et al. Efeito do fósforo e do zinco sobre o crescimento de mudas de freijó (Cordia goeldiana Huber.). Revista de Ciências Agrárias, n.37, p.123-131, 2002.

FERNANDES, A. R. et al. Crescimento e absorção de nutrientes por mudas de Freijó (Cordia goeldiana HUBER) em função de doses de fósforo de zinco. Revista Árvore, v.31, n.4, p.599-608, 2007.
FERNÁNDEZ, J. Q. P. et al. Crescimento de mudas de Mimosa tenuiflora submetidas a diferentes níveis de calagem e doses de fósforo, potássio e enxofre. Revista Árvore, v.20, n.4, p.425-431, 1996.

GOMES, J. M. Parâmetros morfológicos na avaliação da qualidade de mudas de Eucalyptus grandis, produzidas em diferentes tamanhos de tubete e de dosagens de N-P-K. 2001. 126f. Tese (Doutorado em Ciência Florestal) - Universidade Federal de Viçosa, Viçosa, MG, 2001.

GONÇALVES, E. O. et al. Crescimento de mudas de angico-vermelho (Anadenanthera macrocarpa (Benth.) Brenan) sob diferentes doses de macronutrientes. Revista Árvore, v.32, n.6, p.1029-1040, 2008.

LIMA, L. S. H.; FRANCO, E. T. H.; SCHUMACHER, M. V. Crescimento de mudas de Euterpe edulis Martins em resposta a diferentes doses de fósforo. Ciência Florestal, v.19, n.4, p.461-470, 2008.

LORENZI, H. Árvores brasileiras: Manual de identificação e cultivo de plantas arbóreas nativas do Brasil, Nova Odessa: Plantarum, 1992. 352p.

\section{MARISCAL-FLORES, E. J. Potencial} produtivo e alternativas de manejo sustentável de um fragmento de Mata Atlântica secundária, Município de Viçosa, Minas Gerais. 1993. 165f.

Dissertação (Mestrado). Universidade Federal de Viçosa, Viçosa,MG, 1993.

MARQUES, L. S. et al. Crescimento de mudas de Jacaré (Piptadenia gonoacantha J.F. Macbr.) em diferentes tipos de solos e fontes e doses de nitrogênio. Revista Árvore, v.33, n.1, p.81-92, 2009.

MARQUES, V. B. et al. Efeitos de fontes e doses de nitrogênio no crescimento de mudas de sabiá (Mimosa caesalpiniaefolia Benth.). Scientia Forestalis, n.71, p.77-85, 2006.

NEVES, J. C. L. et al. Efeito do alumínio em amostras de dois latossolos sob cerrado sobre o crescimento e absorção de nutrientes de mudas de Eucalyptus spp. Revista Árvore, v.6, n.1, p.17-28, 1982.

R. Árvore, Viçosa-MG, v.34, n.1, p.13-24, 2010 
NEVES, J. C. L. Aspectos nutricionais em mudas de Eucalyptus spp - Tolerância ao alumínio e níveis críticos de fósforo no solo. 1983. 83f. Dissertação (Mestrado em Solos e Nutrição de Plantas) - Universidade Federal de Viçosa, Viçosa, MG, 1983.

PAIVA, H. N.; GOMES, J. M. Viveiros

florestais. 2.ed. Viçosa, MG: Universidade Federal de Viçosa, 2000. 69p. (Cadernos Didáticos, 72).

REIS, M. G. F. et al. Exigências nutricionais de mudas de Dalbergia nigra (Vell.) Fr. Allem (Jacarandá-da-Bahia) produzidas em dois níveis de sombreamento. Revista Árvore, v.21, n.4, p.463-471, 1997.

RESENDE, M.; CURI, N.; SANTANA, D. P. Pedologia e fertilidade do solo: interações e aplicações. Brasília: Ministério da Educação; Lavras: ESAL; Piracicaba: POTAFOS, 1988. 81p.

SOUZA, C. A. M. et al. Crescimento em campo de espécies florestais em diferentes condições de adubações. Ciência Florestal, v.16, n.3, p.243-249, 2006.
SOUZA, P. A.; VENTURIN, N.; MACEDO, R. L. G. Adubação mineral do Ipê-Roxo (Tabebuia impetiginosa). Ciência Florestal, v.16, n.3, p.261-270, 2006.

SOUZA, P. H. et al. Influência da saturação por bases no substrato no crescimento e qualidade de mudas de Machaerium nictitans (Vell.) Benth. Revista Árvore, v.32, n.2, p.193-201, 2008.

TURRENT, F. A. Uso de una matriz mixta para la optimización de cinco a ocho factores controlables de la producción. Chapingo-México: Rama de Suelos, Colégio de Postgraduados, 1979. 65p. (Boletim Técnico, 6)

VALE, F. R. et al. Crescimento radicular de espécies florestais em solo ácido. Pesquisa Agropecuária Brasileira, v.31, n.9, p.606616, 1996.

VENTURIN, R. P. et al. Efeito da relação Ca:Mg do corretivo no desenvolvimento e nutrição mineral de mudas de aroeira (Myracroduon urundeuva Fr. All.). Revista Cerne, v.6, n.1, p.30-39, 1999. 